\title{
ESSENTIAL SUPPLEMENTED LATTICES
}

\author{
HASAN HÜSEYIN ÖKTEN AND AYTEN PEKIN
}

Received 22 February, 2020

\begin{abstract}
Let $L$ be a complete modular lattice. If every essential element of $L$ has a supplement in $L$, then $L$ is called an essential supplemented (or briefly e-supplemented) lattice. In this work some properties of these lattices are investigated. Let $L$ be a complete modular lattice and $1=$ $a_{1} \vee a_{2} \vee \ldots \vee a_{n}$ with $a_{i} \in L(1 \leq i \leq n)$. If $a_{i} / 0$ is e-supplemented for every $i=1,2, \ldots, n$, then $L$ is also e-supplemented. If $L$ is e-supplemented, then $1 / a$ is also e-supplemented for every $a \in L$.
\end{abstract}

2010 Mathematics Subject Classification: 06C05; 06C15

Keywords: lattices, essential elements, small elements, supplemented lattices

\section{INTRODUCTION}

In this paper, every lattice is a complete modular lattice with the smallest element 0 and the greatest element 1 . Let $L$ be a lattice, $x, y \in L$ and $x \leq y$. A sublattice $\{a \in L \mid x \leq a \leq y\}$ is called a quotient sublattice and denoted by $y / x$. An element $a$ of a lattice $L$ is called a complement of $b$ in $L$ if $a \vee b=1$ and $a \wedge b=0$, in this case we denote $1=a \oplus b$ (here we also call $a$ and $b$ are direct summands of $L$ ). $L$ is said to be complemented if each element has at least one complement in $L$. An element $x$ of $L$ is said to be small or superfluous and denoted by $x \ll L$ if $y=1$ for every $y \in L$ such that $x \vee y=1$. Let $L$ be a lattice and $k \in L$. If $t=0$ for very $t \in L$ with $k \wedge t=0$, then $k$ is called an essential element of $L$ and denoted by $k \unlhd L$. The meet of all maximal $(\neq 1)$ elements of a lattice $L$ is called the radical of $L$ and denoted by $r(L)$. If $a \ll L$, then $a \leq r(L)$ holds. An element $a$ of $L$ is called a supplement of $b$ in $L$ if it is minimal for $1=b \vee a$. $a$ is a supplement of $b$ in a lattice $L$ if and only if $1=b \vee a$ and $b \wedge a \ll a / 0$. A lattice $L$ is called a supplemented lattice if every element of $L$ has a supplement in $L$. We say that an element $y$ of $L$ lies above an element $x$ of $L$ if $x \leq y$ and $y \ll 1 / x$. $L$ is said to be hollow if every element distinct from 1 is superfluous in $L$, and $L$ is said to be local if $L$ has the greatest element $(\neq 1)$. We say an element $x \in L$ has ample supplements in $L$ if for every $y \in L$ with $x \vee y=1, x$ has a supplement $z$ in $L$ with $z \leq y$. $L$ is said to be amply supplemented if every element of $L$ has ample supplements in $L$. Let $L$ be a lattice. It is defined $\beta_{*}$ relation on the elements of $L$ by 
$a \beta_{*} b$ with $a, b \in L$ if and only if for each $t \in L$ such that $a \vee t=1$ then $b \vee t=1$ and for each $k \in L$ such that $b \vee k=1$ then $a \vee k=1$.

More informations about (amply) supplemented lattices are in [1, 2, 5, 9]. More results about (amply) supplemented modules are in $[8,12]$. The definition of $\beta_{*}$ relation on lattices and some properties of this relation are in [10]. This relation is a generalization of $\beta^{*}$ relation on modules. The definition of $\beta^{*}$ relation on modules and some properties of this relation are in [4].

Lemma 1. Let $L$ be a lattice and $a, b, c \in L$ with $a \leq b$. If $c$ is a supplement of $b$ in $L$, then $a \vee c$ is a supplement of $b$ in $1 / a$.

Proof. See [3, Lemma 1].

Definition 1. Let $L$ be a lattice and $k$ be a maximal $(\neq 1)$ element of $L$. If $k \unlhd L$, then $k$ is called a g-maximal element of $L$. The meet of all g-maximal elements of $L$ is called the g-radical of $L$ and denoted by $r_{g}(L)$. If $L$ have not any g-maximal elements, then we call $r_{g}(L)=1$.

The g-radical of any lattice is a generalization of the generalized radical of any module. The definition of the generalized radical of any module and some properties of this concept are in $[6,7]$.

Corollary 1. Let $L$ be a lattice. Then $r(L) \leq r_{g}(L)$.

Proof. Clear from definitions.

Lemma 2. Let $L$ be a lattice and $x \in L$. Then $r_{g}(x / 0) \leq r_{g}(L)$.

Proof. Let $k$ be any g-maximal element of $L$. If $x \leq k$, then $r_{g}(x / 0) \leq k$. If $x \not k k$, we can easily see that $x \wedge k$ is a g-maximal element of $x / 0$ and hence $r_{g}(x / 0) \leq k$. Therefore, $r_{g}(x / 0) \leq r_{g}(L)$.

\section{ESSENTIAL SUPPLEMENTED LATTICES}

Definition 2. Let $L$ be a lattice. If every essential element of $L$ has a supplement in $L$, then $L$ is called an essential supplemented (or briefly e-supplemented) lattice.

This concept is a generalization of an essential supplemented module. The definition of essential supplemented modules and some properties of these modules are in [11].

It is clear that every supplemented lattice is e-supplemented. Hollow and local lattices are e-supplemented.

Proposition 1. Let $L$ be an e-supplemented lattice. If every element of $L$ with distinct from 0 is essential in $L$, then $L$ is supplemented.

Proof. Clear from definitions. 
Definition 3. Let $L$ be a lattice and $a \in L$. If $a$ is a supplement of an essential element in $L$, then $a$ is called an e-supplement element in $L$.

Proposition 2. Let $L$ be a lattice. If every essential element of $L$ is e-supplement in $L$, then $L$ is e-supplemented.

Proof. Let $x \unlhd L$. By hypothesis $x$ is an e-supplement element in $L$ and there exists $y \unlhd L$ such that $x$ is a supplement of $y$ in $L$. Here $1=y \vee x$ and $y \wedge x \ll x / 0$. Since $y \unlhd L$, by hypothesis, $y$ is an e-supplement element in $L$. Since $x \wedge y \ll L$ and $y$ is a supplement element in $L$, by [9, Lemma 10], $x \wedge y \ll y / 0$ and $y$ is a supplement of $x$ in $L$. Hence $L$ is e-supplemented.

Proposition 3. Let $L$ be a lattice and a be an e-supplement element in $L$. Then $r_{g}(a / 0)=a \wedge r_{g}(L)$.

Proof. By Lemma 2, $r_{g}(a / 0) \leq a \wedge r_{g}(L)$ holds. Since $a$ is a e-supplement element in $L$, there exists an essential element $x$ of $L$ such that $a$ is a supplement of $x$ in $L$. Here $1=x \vee a$ and $x \wedge a \ll a / 0$. By Corollary $1, x \wedge a \leq r(a / 0) \leq r_{g}(a / 0)$ hold. Let $k$ be any g-maximal element of $a / 0$. Here $x \wedge a \leq r_{g}(a / 0) \leq k$. Since $\frac{1}{x \vee k}=\frac{x \vee k \vee a}{x \vee k} \cong \frac{a}{a \wedge(x \vee k)}=\frac{a}{(x \wedge a) \vee k}=\frac{a}{k}$ and $x \vee k \unlhd L, x \vee k$ is a g-maximal element of $L$ and hence $r_{g}(L) \leq x \vee k$. This case $a \wedge r_{g}(L) \leq a \wedge(x \vee k)=(x \wedge a) \vee k=k$. Therefore, $a \wedge r_{g}(L) \leq r_{g}(a / 0)$ and since $r_{g}(a / 0) \leq a \wedge r_{g}(L), r_{g}(a / 0)=a \wedge r_{g}(L)$.

Proposition 4. Let $L$ be an essential supplemented lattice. Then $1 / r(L)$ doesn" $t$ have any essential element with distinct from 1.

Proof. Let $x$ be any essential element of $1 / r(L)$. Then we clearly see that $x$ is an essential element of $L$. Since $L$ is essential supplemented, $x$ has a supplement $y$ in $L$. Here $1=x \vee y$ and $x \wedge y \ll y / 0$. Since $x \wedge y \ll y / 0, x \wedge y \leq r(L)$. Here $1=x \vee y=$ $x \vee y \vee r(L)$ and $x \wedge(y \vee r(L))=(x \wedge y) \vee r(L)=r(L)$. Hence $1=x \oplus(y \vee r(L))$ in $1 / r(L)$ and since $x \unlhd 1 / r(L), x=1$.

Lemma 3. Let $L$ be a lattice, $x \unlhd L$ and $a \in L$. If $x \vee$ a has a supplement in $L$ and $a / 0$ essential supplemented, then $x$ has a supplement in $L$.

Proof. Let $y$ be a supplement of $x \vee a$ in $L$. Then $1=x \vee a \vee y$ and $(x \vee a) \wedge y \ll$ $y / 0$. Since $x \unlhd L, x \vee y \unlhd L$ and hence $(x \vee y) \wedge a \unlhd a / 0$. Since $a / 0$ is essential supplemented, $(x \vee y) \wedge a$ has a supplement $z$ in $a / 0$. Here $a=[(x \vee y) \wedge a] \vee z$ and $(x \vee y) \wedge z=(x \vee y) \wedge a \wedge z \ll z / 0$. Following these we have $1=x \vee a \vee y=x \vee y \vee$ $[(x \vee y) \wedge a] \vee z=x \vee y \vee z$ and $x \wedge(y \vee z) \leq[(x \vee y) \wedge z] \vee[(x \vee z) \wedge y] \leq[(x \vee y) \wedge z] \vee$ $[(x \vee a) \wedge y] \ll(y \vee z) / 0$. Hence $y \vee z$ is a supplement of $x$ in $L$.

Lemma 4. Let $L$ be a lattice and $1=a \vee b$ with $a, b \in L$. If $a / 0$ and $b / 0$ are e-supplemented, then $L$ is also e-supplemented.

Proof. Let $x \unlhd L$. Then 0 is a supplement of $x \vee a \vee b$ in $L$. Since $b / 0$ is esupplemented and $x \vee a \unlhd L$, by Lemma 3, $x \vee a$ has a supplement in $L$ and since 
$a / 0$ is e-supplemented and $x \unlhd L$, again by Lemma $3, x$ has a supplement in $L$. Hence $L$ is e-supplemented.

Corollary 2. Let $L$ be a lattice and $1=a_{1} \vee a_{2} \vee \ldots \vee a_{n}$ with $a_{i} \in L(1 \leq i \leq n)$. If $a_{i} / 0$ is e-supplemented for every $i=1,2, \ldots, n$, then $L$ is also e-supplemented.

Proof. Clear from Lemma 4.

Lemma 5. Let $L$ be an e-supplemented lattice and $a \in L$. Then $1 / a$ is also $e$ supplemented.

Proof. Let $x$ be any essential element of $1 / a$. Then $x \unlhd L$ and since $L$ is esupplemented, $x$ has a supplement $y$ in $L$. By Lemma $1, y \vee a$ is a supplement of $x$ in $1 / a$. Hence $1 / a$ is e-supplemented.

Corollary 3. Let $L$ be an e-supplemented lattice. Then a $/ 0$ is e-supplemented for every direct summand a of $L$.

Proof. Since $a$ is a direct summand of $L$, there exists $b \in L$ such that $1=a \oplus b$. By Lemma $5,1 / b$ is e-supplemented. Then by $\frac{1}{b}=\frac{a \vee b}{b} \cong \frac{a}{a \wedge b}=\frac{a}{0}, a / 0$ is also esupplemented.

Lemma 6. Let $L$ be a lattice. If every essential element of $L$ is $\beta_{*}$ equivalent to an e-supplement element in $L$, then $L$ is e-supplemented.

Proof. Let $x \unlhd L$. By hypothesis, there exists an e-supplement element $y$ in $L$ such that $x \beta_{*} y$. Since $y$ is an e-supplement element in $L$, there exists $z \unlhd L$ such that $y$ is a supplement of $z$ in $L$. By hypothesis, there exists an e-supplement element $a$ in $L$ such that $z \beta_{*} a$. Since $y$ is a supplement of $z$ in $L$ and $z \beta_{*} a$, by [10, Theorem 4 (1)], $y$ is a supplement of $a$ in $L$. Here $1=a \vee y$ and $a \wedge y \ll y / 0$. Since $a$ is a supplement element in $L$, by [9, Lemma 10], $a \wedge y \ll a / 0$ and $a$ is a supplement of $y$ in $L$. Since $x \beta_{*} y$, by [10, Theorem 4 (1)], $a$ is a supplement of $x$ in $L$. Hence $L$ is e-supplemented.

Corollary 4. Let $L$ be a lattice. If every essential element of $L$ lies above an e-supplement element in $L$, then $L$ is e-supplemented.

Proof. Clear from [10, Theorem 3] and Lemma 6.

Definition 4. Let $L$ be a lattice. If every essential element of $L$ with distinct from 1 is small in $L$ or $L$ have no essential elements with distinct from 1 , then $L$ is called a ehollow lattice. If $L$ has an essential element $c \neq 1$ such that $k \leq c$ for every $1 \neq k \unlhd L$, then $L$ is called an e-local lattice (here $k$ is called the greatest essential element $(\neq 1)$ of $L$ ).

Clearly we can see that every hollow lattice is e-hollow. But the converse is not true in general (See Example 1).

Proposition 5. Every e-hollow lattice is e-supplemented. 
Proof. Let $L$ be an e-hollow lattice. Then 1 is a supplement of every essential element of $L$ with distinct from 1 and 0 is a supplement of 1 in $L$. Hence $L$ is esupplemented.

Proposition 6. Let $L$ be a lattice with $r(L) \unlhd L$ and $r(L) \neq 1$. If $L$ is e-hollow, then $L$ is e-local.

Proof. Let $x \unlhd L$ and $x \neq 1$. Since $L$ is e-hollow, $x \ll L$ and $x \leq r(L)$. By hypothesis, $r(L) \neq 1$. Hence $r(L)$ is the greatest essential element $(\neq 1)$ of $L$ and $L$ is e-local.

Example 1. Consider the lattice $L=\{0, a, b, 1\}$ given by the following diagram.

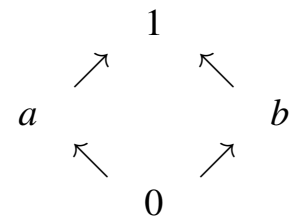

Then $L$ is e-hollow but not hollow. Here $r(L)=0 \neq 1=r_{g}(L)$ hold.

Example 2. Consider the lattice $L=\{0, a, b, c, 1\}$ given by the following diagram.

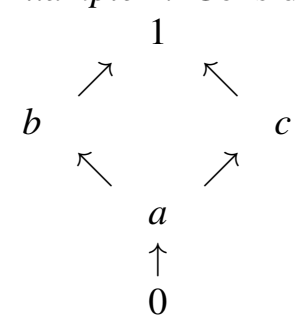

Then $L$ is e-supplemented but not e-hollow.

\section{REFERENCES}

[1] R. Alizade and S. E. Toksoy, "Cofinitely weak supplemented lattices," Indian J. Pure Appl. Math., vol. 40, no. 5, pp. 337-346, 2009.

[2] R. Alizade and S. E. Toksoy, "Cofinitely supplemented modular lattices," Arab. J. Sci. Eng., vol. 36, no. 6, pp. 919-923, 2011, doi: 10.1007/s13369-011-0095-z. [Online]. Available: https://doi.org/10.1007/s13369-011-0095-z

[3] Ç. Biçer and C. Nebiyev, " $\oplus-$-supplemented lattices," Arab. J. Sci. Eng., vol. 20, no. 2, pp. $773-$ 780, 2019, doi: 10.18514/MMN.2019.2806.

[4] G. F. Birkenmeier, F. Takil Mutlu, C. Nebiyev, N. Sokmez, and A. Tercan, "Goldie*-supplemented modules," Glasg. Math. J., vol. 52, no. A, pp. 41-52, 2010, doi: 10.1017/S0017089510000212. [Online]. Available: https://doi.org/10.1017/S0017089510000212

[5] G. Călugăreanu, Lattice concepts of module theory, ser. Kluwer Texts in the Mathematical Sciences. Kluwer Academic Publishers, Dordrecht, 2000, vol. 22. [Online]. Available: https://doi.org/10.1007/978-94-015-9588-9. doi: 10.1007/978-94-015-9588-9

[6] B. Koşar, C. Nebiyev, and N. Sökmez, " $g$-supplemented modules," Ukrainian Math. J., vol. 67, no. 6, pp. 975-980, 2015, translation of Ukraïn. Mat. Zh. 67 (2015), no. 6, 861-864, doi: 10.1007/s11253-015-1127-8. [Online]. Available: https://doi.org/10.1007/s11253-015-1127-8 
[7] B. Koşar, C. Nebiyev, and A. Pekin, "A generalization of g-supplemented modules," Miskolc Math. Notes, vol. 20, no. 1, pp. 345-352, 2019, doi: 10.18514/mmn.2019.2586. [Online]. Available: https://doi.org/10.18514/mmn.2019.2586

[8] C. Nebiyev and A. Pancar, "On supplement submodules," Ukrainian Math. J., vol. 65, no. 7, pp. 1071-1078, 2013, doi: 10.1007/s11253-013-0842-2. [Online]. Available: https://doi.org/10.1007/s11253-013-0842-2

[9] C. Nebiyev, "On supplement elements in lattices," Miskolc Math. Notes, vol. 20, no. 1, pp. 441$449,2019$.

[10] C. Nebiyev and H. H. Ökten, " $\beta_{*}$ relation on lattices," Miskolc Math. Notes, vol. 18, no. 2, pp. 993-999, 2017, doi: 10.18514/mmn.2017.1782. [Online]. Available: https: //doi.org/10.18514/mmn.2017.1782

[11] C. Nebiyev, H. H. Ökten, and A. Pekin, "Essential supplemented modules," International Journal of Pure and Applied Mathematics, vol. 120, no. 2, pp. 253-257, 2018, doi: 10.12732/ijpam.v120i2.9.

[12] R. Wisbauer, Foundations of module and ring theory, ser. Algebra, Logic and Applications. Gordon and Breach Science Publishers, Philadelphia, PA, 1991, vol. 3, a handbook for study and research.

Authors' addresses

Hasan Hüseyin Ökten

Amasya University, Technical Sciences Vocational School, Amasya, Turkey

E-mail address: hokten@gmail.com

Ayten Pekin

Istanbul University, Department of Mathematics, Vezneciler, Istanbul, Turkey

E-mail address: aypekin@istanbul.edu.tr 